\title{
The treatment of gambling addiction-why important to provide a different approach than chemical addiction
}

Volume 7 Issue 6 - 2017

\section{Mini review}

One of the principle concerns facing all Therapists is how to optimally match treatment appropriately to the clinical needs of the client and the requirements of the treatment plan. However matching people to the appropriate level of clinical care is more complex than it might first appear and providing treatment is a blend of science and art. For example, the same treatment, may not be the same treatment for all people, likewise treatment that is suitable with Chemical Addiction may not be suitable when working with Gambling Addictions. Pathological Gamblers often experience their problems differently even from other gamblers; Within the field of gambling disorders some disorders take different courses for some individuals compared with others. Similarly, individuals with a gambling disorder experience differing degrees of infirmity at different points during the development of their illness. As such, these individuals are likely to either need differlng intensities of treatment or different treatment approaches altogether. The first piece of evidence to support these ideas comes from prevalence rates. Certain groups seem to be more vulnerable to developing problem/pathological gambling than other groups. The second piece of evidence comes from research suggesting a high comorbidity rate with a number of mental illnesses. The third research thread suggests that individuals with the same diagnosis might be at different stages/points in their illness and consequently need a different type of treatment. It is worth noting that most gambling studies on the relationship between gambling and other psychiatric conditions have been focused on substance use and mood disorders (e.g. Fiegelman, Wallisch \& Leisieur 1998 Welte, Barnes) Crockford and el-Guebaly report that between $25 \%$ and $63 \%$ of pathological gamblers meet criteria for a substance use disorder in their lifetime. Correspondingly, $9 \%$ to $16 \%$ of clients with a substance use disorder are also found to be probable pathological gamblers. When more than one substance is abused, the prevalence and severity of pathological gambling is increased as compared to individual who abuse only one drug. It is important that therapists working with Gambling Addiction provide a diverse approach. To illustrate, therapists can frame gambling disorders as a excessive attachment to money or diminished cognitive capacity. Alternatively, gambling disorders can be framed as the result of deficient ego functions (e.g. ego defences, self - esteem, affect regulation and management, self-care and object relations). When therapists and clients talk about using gambling, drugs, food, or alcohol to numb the pain, keep them company, relieve anxiety or fill up an empty feeling, they are using Pyschodynamiclauguage. In contrast, many therapists use a behavioural frame to make sense of their client's experience. When addiction treatment specialists focus on the risks that clients face by being in gambling settings, on how hard clients must work to break their habit, or how stepping into a casino 'triggers' a train of impulsive thoughts, feelings, and behaviours, therapists are featuring a behavioural story to frame the problem.

\author{
Mark Dempster \\ Director, Mark Dempster Counselling, UK
}

Correspondence: Mark Dempster, Director, Mark Dempster Counselling, 7 Harley Street, London, WI G 9QY, UK, Tel 020 3239 706I,Email mark@maekdempstercounselling.com

Received: April 06, 2016 | Published: June 07, 2017
Gambling addiction differs from chemical addiction in one central perspective; Gamblers are fundamentally preoccupied with money and credit. Drug addicts/alcoholis are preoccupied with the chemical. The Gambler's drug of choice is money therefore it is essential to address their financial obligations and responsibilities during treatment. By reducing these very real and pressing problems, treatment can reduce the stress and anxiety associated with financial debt. I have found it useful in my provide financial management counselling as a key component in treatment, this includes on occasion 1- Identifying assets and income 2- Creating and maintaining a spending plan 3shifting all financial control to the non gambling family member 4repaying debts 5 - Investing wisely.

Therapists can use the disease model with those clients most familiar and comfortable with understanding their 'problem' from a sickness or illness frame. Any single client-therapist pair may use different components of these or other stories during any given treatment encounter. The prescriptive approach recognizes that a clients perception of his or her disorder will determine when, how and to what treatment provider, if any, he or she will go. Understanding a client's perception of his or her gambling problem permits the therapist to negotiate and navigate treatment interventions. Rather than one conceptual frame, therapists can learn how to access and then to assess the clients view of addiction. While it might be possible to impose a clinical model on some clients, most bring their own view of gambling and addiction with them when they enter treatment. When the therapist and the clients see things similarly, treatment can go well. However most observers recognise that the majority of intemperate gamblers find it difficult to engage in treatment. Historically this has been a client problem: Many treatment specialists blame the client and the disease of denial. More likely, however, this situation results when clients views do not match the therapists opinion Research pertaining to the treatment of gambling disorders is in its infancy. It is likely that there is no single best treatment for gambling disorders. It is suggested that the symptoms associated with pathological gambling 
reflect a complex syndrome instead of a single disorder. Treatments for gambling disorders are promising. The treatment strategies that are widely used often have their roots in other addictive disorders. However the clinical practice that of other addictive disorders also might be informed by the techniques that have evolved in treating gambling.

\section{Acknowledgments}

None.

\section{Conflicts of interest}

Author declares there are no conflicts of interest.

\section{Funding}

None. 